\title{
On the Difference of Scaling Properties for Temperature and Precipitation over China
}

\author{
Lei Jiang, ${ }^{1}$ Liqing Zhao, ${ }^{1}$ and Zihao Zhao ${ }^{2}$ \\ ${ }^{1}$ School of Marine Sciences, Nanjing University of Information Science and Technology, Nanjing 210044, China \\ ${ }^{2}$ School of Applied Meteorology, Nanjing University of Information Science and Technology, Nanjing 210044, China \\ Correspondence should be addressed to Lei Jiang; jianglei@nuist.edu.cn
}

Received 14 August 2017; Revised 4 November 2017; Accepted 5 December 2017; Published 20 December 2017

Academic Editor: Jorge E. Gonzalez

Copyright (c) 2017 Lei Jiang et al. This is an open access article distributed under the Creative Commons Attribution License, which permits unrestricted use, distribution, and reproduction in any medium, provided the original work is properly cited.

\begin{abstract}
The daily air temperature and precipitation records of four meteorological observation stations over China are used to investigate the differences of scaling property employing the detrended fluctuation analysis (DFA) method. The results show that the values in DFA-exponent for temperature are higher than those for precipitation compared by different orders DFA1-3. A 95\% significance test is also applied to verify LRCs by resampling the temperature and precipitation records 10000 times in Beijing. The values of scaling exponent from original temperature and precipitation records are larger than the upper range value of the interval threshold after shuffling the data records, which implies there are positive LRCs. For temperature records, the value of scaling exponent calculated by FA is greater than those by DFA1-3 at all four stations. This indicates that the FA curve overestimates the scaling behavior due to the effect of trends. By contrast, the values of scaling exponent in precipitation are almost the same by using FA and DFA1-3 for all time scales, respectively. Furthermore, there are crossovers on short time scales in different orders DFA1-3 for the temperature records, while the slopes keep almost consistent on all time scales for the precipitation records.
\end{abstract}

\section{Introduction}

Variations in air temperature and precipitation records are important indicators of climate change. Some studies have exhibited the variation trends of temperature and precipitation at different spatial-temporal scales [1]. Variable persistence represents memory characteristics within data sets at various time scales. The temperature and precipitation time series display different self-similar structures and long range correlations (LRCs). The persistence at various time scales is governed by different physical processes and sometimes even affected by the trends. There is little research on the difference of scaling properties for temperature and precipitation time series over China.

Temperature and precipitation time series show obvious nonstationary characteristics in climate system. In fact, it is not feasible to detect intrinsic dynamic properties of variables due to nonstationarity and nonlinearity by using some traditional approaches, such as the power spectrum and correlation analysis. Therefore, the detrended fluctuation analysis (DFA) method with different orders is applied to investigate scaling behaviors, which may eliminate the data trends often masked by nonstationarities.

Peng et al. successfully developed the DFA method [2]. Since then, many studies have used the DFA method in atmospheric temperature [3-8], cloud breaking [9], wind speed [10], relative humidity [11, 12], air pollution [13], global tropopause [14], ozone variations [15], sea surface temperature [16-18], and so on. In China, different fractal characteristics of air temperature and ground surface temperature have been also studied [19]. Jiang et al. found that most of the values of ground surface temperature in DFAexponent are higher values than those of air temperature over China [20]. Meanwhile, Jiang et al. also studied LRCs of precipitation over China [21]. The precipitation time series, as an important fluctuation indicator in climate system, exhibits 
weak persistence characteristics. A case study of temperature and precipitation records can provide a basis and deepen our understanding for examining seasonal, interannual, and interdecadal variability and trends over China.

The first aim of this paper is to study the differences of LRCs for temperature and precipitation time series over China. The paper is organized as follows. In Section 2, the acquisition of the temperature and precipitation records and the FA and DFA methods are described. In Section 3, the difference of LRCs for temperature and precipitation is investigated and compared using FA and DFA over China. The conclusion and discussions are summarized and drawn in Section 4.

\section{Data and Methods}

2.1. Data Records. The daily air temperature and precipitation data records used in this paper come from the Chinese National Meteorological Information Center. Most of data length is from 1951 to 2009. The long data records have been investigated in various aspects. Yuan et al. used the DFA method to analyze the LRCs of five kinds of daily temperature records and found that there are power law correlated characteristics [22]. The high-quality air temperature and precipitation records are also applied to detect the persistence characteristics and trends at various time scales over China $[20,21,23]$. We removed the seasonal cycles from the raw time series to obtain the air temperature and precipitation anomaly records. The anomaly records can be expressed by $\Delta x_{i}=x_{i}-\left\langle x_{i}\right\rangle_{d}, i=1 \cdots N$, where $x_{i}$ denotes the raw data, $N$ is the data length, and $\left\langle x_{i}\right\rangle_{d}$ represents the mean value for a given calendar day.

2.2. Methodology. Compared with the traditional approaches such as the power spectrum technique and autocorrelation functions analysis, detrended fluctuation analysis systematically eliminates the trends of recorded data and reveals intrinsic dynamic characteristics often masked by nonstationarities. The DFA methodology has been successfully applied in detecting long range correlations of data records such as temperature time series.

Such technique was established by Peng et al. [2] and extended by Bunde et al. [24] and Kantelhardt et al. [25]. Three key steps are described in the DFA method.

(1) Obtain the profile by integrating the air temperature and precipitation anomaly records with $N$ samples:

$$
y(i)=\sum_{k=1}^{i} \Delta x_{k}, \quad i=1, \ldots, N
$$

where $y(i)$ is the profile, $\Delta x_{k}$ is deseasoned records, and $N$ is data length.

(2) Divide the profile into nonoverlapping segments of equal length $s$, indexed by $k=1, \ldots, N_{s}$ with $N_{s} \equiv$ $[N / s]$. A least-square fit $y_{v}(k)$ is used to determine the local polynomial trend in each segment.

$$
y^{\prime}(i)=y(i)-y_{v}(i)
$$

In each of these segments, the local trend $y_{v}(k)$ is calculated by least-square fits in the interval. In the firstorder detrended fluctuation analysis (DFA1), the detrended variability $F(s)$ can be determined from the linear fit of the profile for each window $s$. The possible linear trends are eliminated by this step. In DFA2, possible linear and parabolic trends are removed by subtracting quadratic fits of the profile. Likewise, in DFAn, possible $n$ order trends are eliminated in the profile. However, in FA, any kind of trend is retained in the profile.

(3) The fluctuation function $F^{(n)}(s)$ is calculated by the profile subtracting the local polynomial fit in each segment length $s$ :

$$
F^{(n)}(s)=\sqrt{\frac{1}{2 N_{s}} \sum_{k=1}^{2 N_{s}}\left[y(k)-y_{s}(k)\right]^{2}} .
$$

There is double logarithmic linear relationship $F(s) \sim s^{\alpha}$ between the fluctuation function $F(s)$ and time window $s$. This represents the correlation characteristic can be depicted by the scaling exponent $\alpha$. The data signal exhibits random behaviors for $\alpha=0.5$, positive LRCs for $\alpha>0.5$, and anticorrelated behaviors for $\alpha<0.5$, respectively.

In different order $n$ of DFA, the $n$ th-order trend of time series is eliminated by subtracting polynomial fittings such as linear, quadratic, cubic, and higher order corresponding to DFA1, DFA2, DFA3, and higher order DFA. DFAn removes $n$-order trend of the profile and $n-1$-order trend of the original data records. The trend strength of temperature and precipitation time series can be estimated by using different orders of DFA.

\section{Results}

The temporal evolution of the daily temperature and precipitation anomaly records is shown in Figure 1 at four selected weather stations over China. The four stations include Beijing, Langfang, Tangshan, and Baoding. The temperature and precipitation anomaly records exhibit irregular high frequency variations for all weather stations. The fluctuations of the precipitation anomaly records are more intense than that for the temperature anomaly records. The probability of extreme events for precipitation is higher than that for temperature due to the large fluctuations.

See Figure 2; profiles of temperature and precipitation anomaly time series vary with the totally different tendency. There are obvious persistence characteristics of temperature anomaly time series at four stations in Figure 2(a). The temperature anomaly records firstly decrease below the value 0 and then gradually increase to 0 and show a similar letter shape "v" and positive linear trends. These indicate that the persistence characteristics of temperature profile at such four stations are stronger than those of precipitation records shown in Figure 2(b).

To characterize the LRCs, the FA and DFA methods are applied to analyze the daily temperature and precipitation anomaly records of four weather stations over China. Figure 3 exhibits double log plots of the FA and DFA1-3 results 


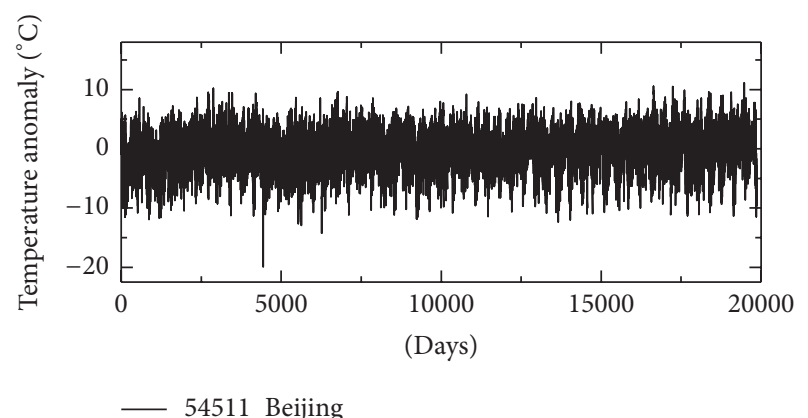

(a)

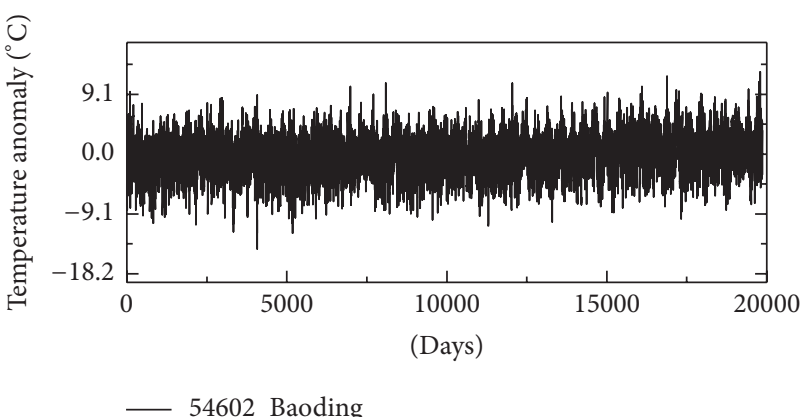

(b)



(c)

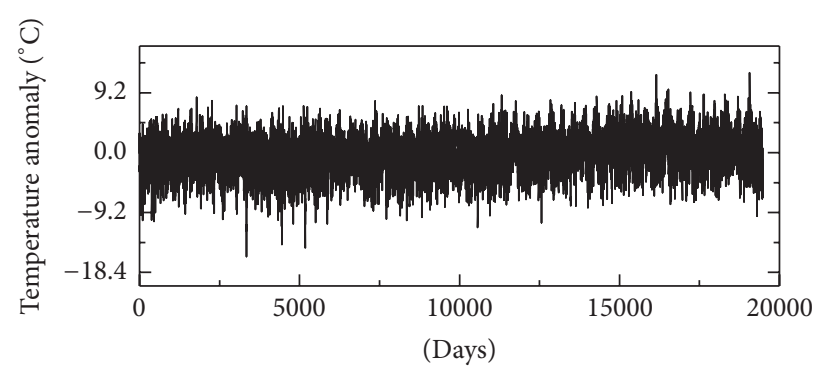

— 54518_Langfang

(d)



(e)

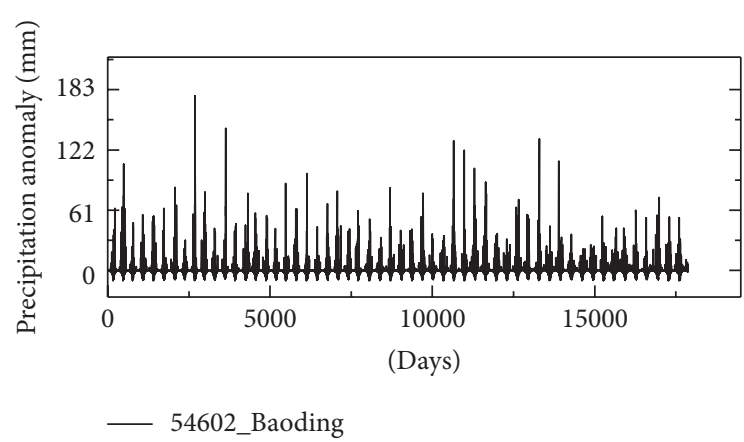

(f)



(g)

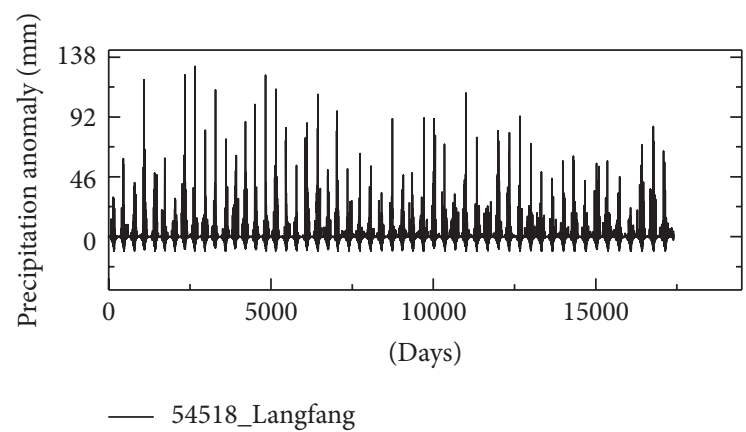

(h)

FIGURE 1: Temporal evolution of the temperature and precipitation anomaly records of four meteorological observation stations over China. ((a) and (e)) Station of Beijing. ((b) and (f)) Station of Baoding. ((c) and (g)) Station of Tangshan. ((d) and (h)) Station of Langfang. 


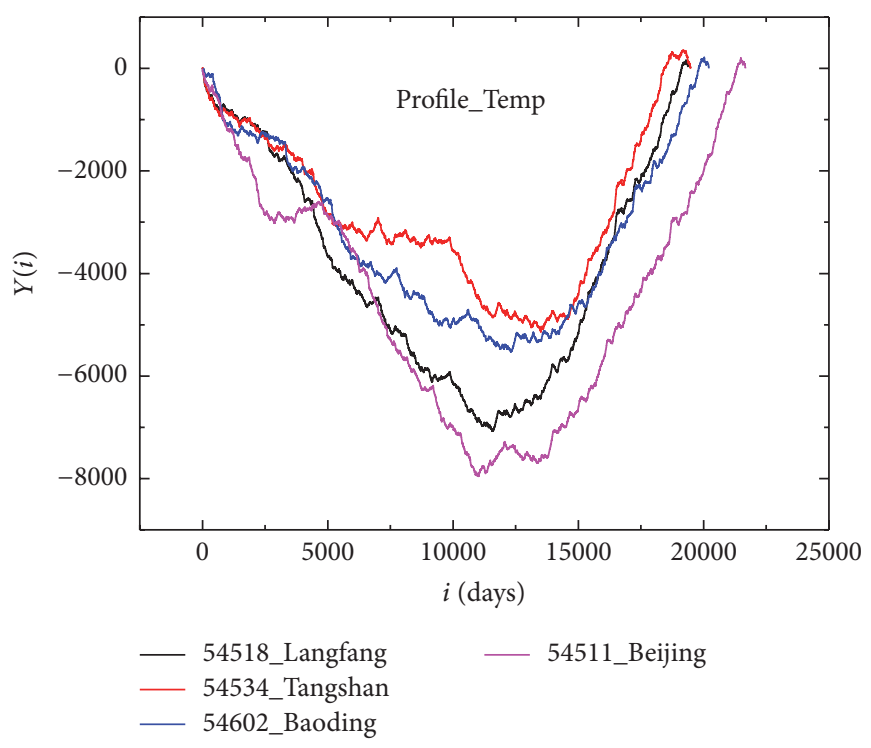

(a)



(b)

Figure 2: (a) Profiles of the temperature anomaly records at four weather stations. (b) Profiles of the precipitation anomaly records.

for temperature anomaly time series. The fluctuations of temperature display the characteristics of long range power law correlations across all four stations. For the temperature anomaly records, the scaling exponent obtained from the FA method is greater than the values from the DFA1-3 at whole time scales. Large value in scaling exponent indicates the results using the FA method may overestimate the LRCs due to the existence of trends in temperature time series. Urbanization as one of the factors might affect the significant difference of scaling property in temperature at short time scales over China.

Similarly, long range power law relationships of precipitation anomaly time series are shown in Figure 4. For the precipitation anomaly records, the curves are straight lines with approximately the same scaling exponent across all time scales using FA and DFA1-3, implying that there are no obvious nonlinear trends. It seems that the scaling behavior of precipitation over China is almost not affected across time scales. There are weak LRCs in precipitation anomaly time series.

The values of scaling exponent in temperature anomaly records are significantly higher than those in precipitation. There are diversity characteristics for the curves in Figure 3 compared with Figure 4. An interesting finding is that the FA curves in temperature time series are approximately a straight line across all time scales at all weather stations. Additionally, the slope of FA curve seems to be consistent with the slopes of DFA1-3 curves at short time scales. However, the slopes of temperature anomaly records are significantly different at short time scales using the DFAl-3 method.

Frequently, the data records do not show the same scaling behaviors for all time scales and their temporal correlation characteristics are different. So one or one more crossovers are detected by the DFA method [26]. For instance, the longterm persistence of data records might become stronger on small time scales than that on large time scales. Moreover, the crossovers might exist in the fluctuation functions with different orders of detrending due to the effect of trends. In addition, there are crossovers for all DFAl-3 curves of temperature at short time scales. Crossovers in the DFA1-3 curves imply that there are different correlation characteristics at various time windows. The existence of the linear trend may be a reason why the value of scaling exponent in the FA curve is higher than in the DFAl-3 curves.

For all four meteorological stations, there are similar persistence characteristics of temperature and precipitation anomaly records, respectively. Next, Beijing, as a representative observation station, is used to analyze LRCs of data records. To further confirm our findings, the slopes of DFAl-3 for temperature records in Beijing are separated by different time windows in Figure 5. The values of scaling exponent gradually decrease with the increase of the order $n$ of DFA at short time scales. The smaller the time scales are, the greater the influence is. However, the values of scaling exponent have small changes in long time scales to some extent. This indicates that local trend effects on LRCs of temperature in Beijing hold within certain time ranges. There are nearly similar slopes for three different orders DFA1-3 in temperature at long time scales.

A numerical test is applied to eliminate the trends and correlations by resampling the temperature and precipitation records 10000 times in order to verify LRCs at different time ranges in Beijing. Figure 6 shows the probability density distribution of scaling exponent by resampling the temperature and precipitation records inside a loop for 10000 times 

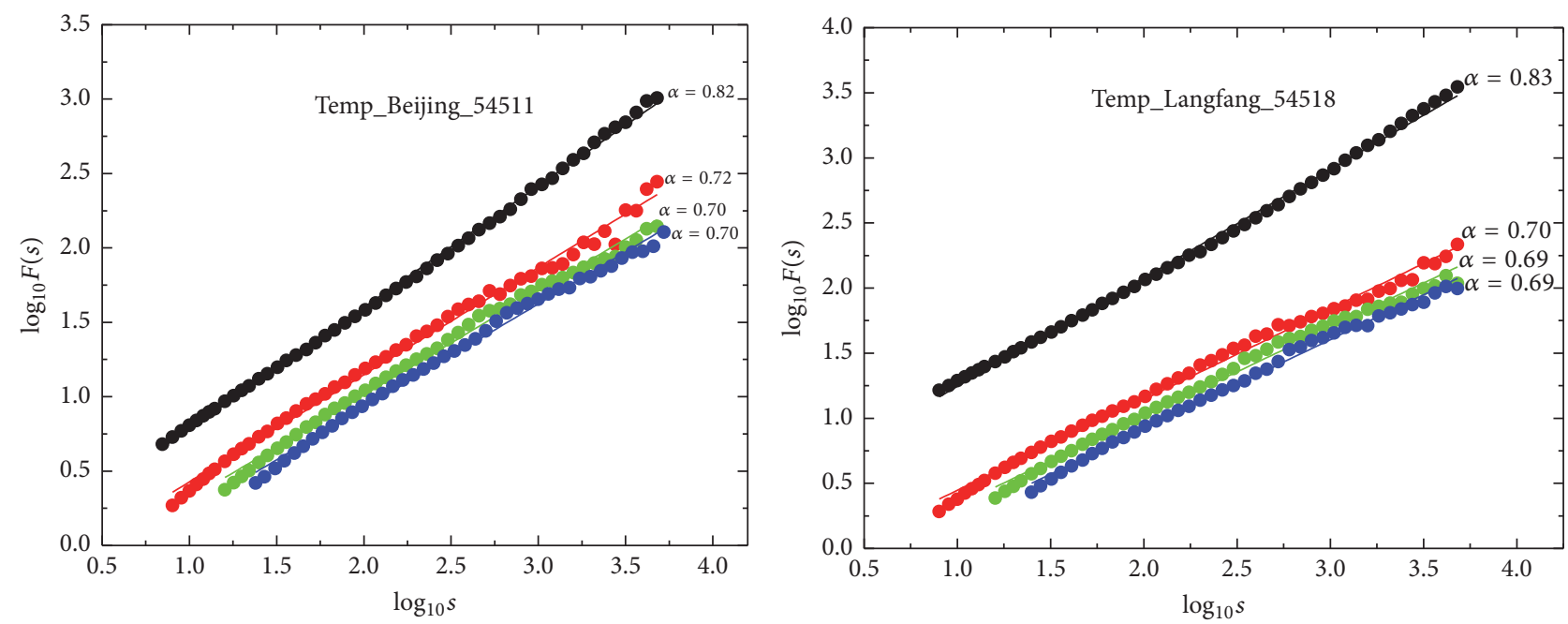

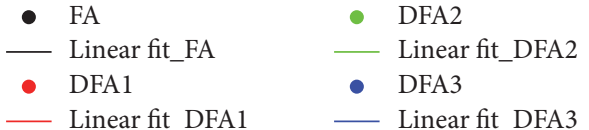

(a)

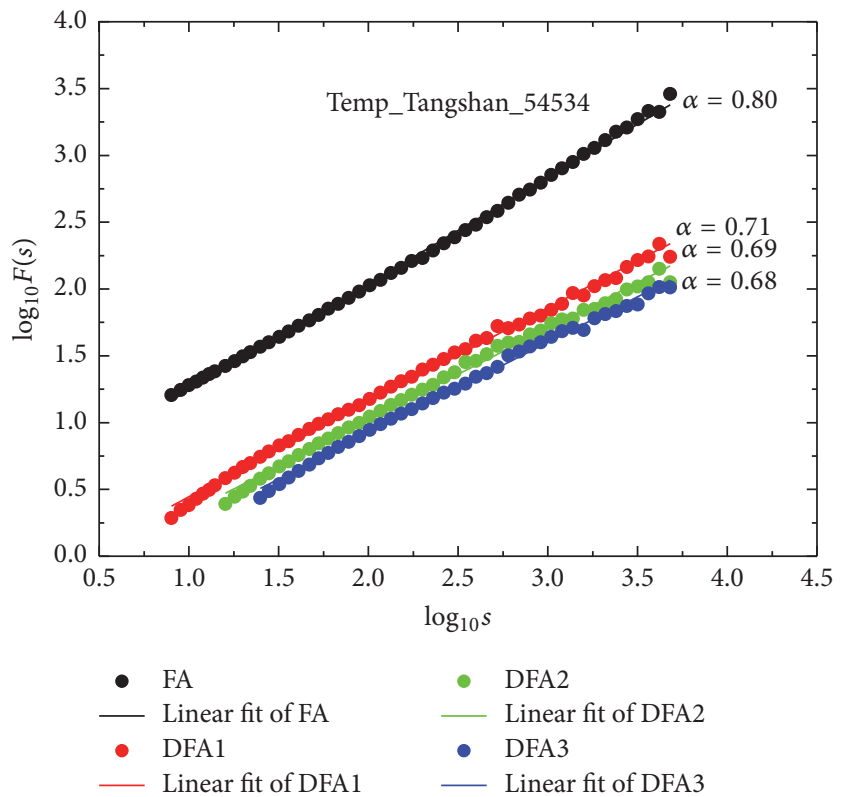

(c)

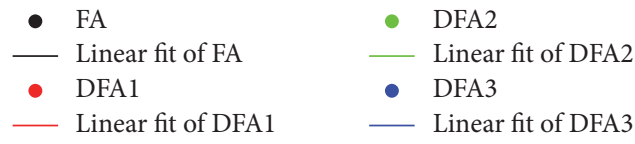

(b)

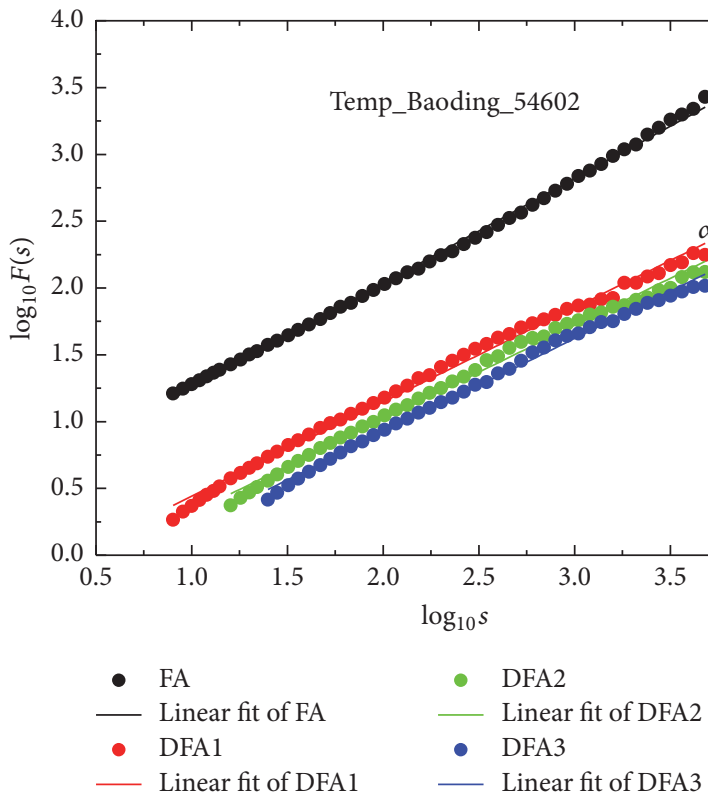

(d)

Figure 3: Log-log plots of power law relationship in temperature anomaly records using FA and DFA1-3 (black, red, green, and blue solid circles, resp.) at four stations. (a) Beijing. (b) Langfang. (c) Tangshan. (d) Baoding. Solid lines are linear fits.

applying the DFA2 method. Figure 6(a) exhibits the frequency distributions of the scaling exponent for temperature. The range of the interval threshold is from 0.47 to 0.53 for temperature records at $95 \%$ significant level. Similarly, the interval threshold is the same range for precipitation records shown in Figure 6(b). The maximum frequencies of scaling exponent in shuffled temperature and precipitation records are centered on 0.50 and take on the normal distributions.
The value $\alpha=0.70$ for temperature and the value $\alpha=0.57$ for precipitation in Beijing are significantly larger than the upper threshold of scaling exponent $\alpha=0.53$ at $95 \%$ confidence level. Therefore, there are the LRCs for temperature and precipitation records in Beijing. Moreover, the Gauss fits of scaling exponent in temperature are almost consistent with that in precipitation shown in Figure 6(c). The Gauss fit curve in precipitation is slightly higher toward the maximum value 

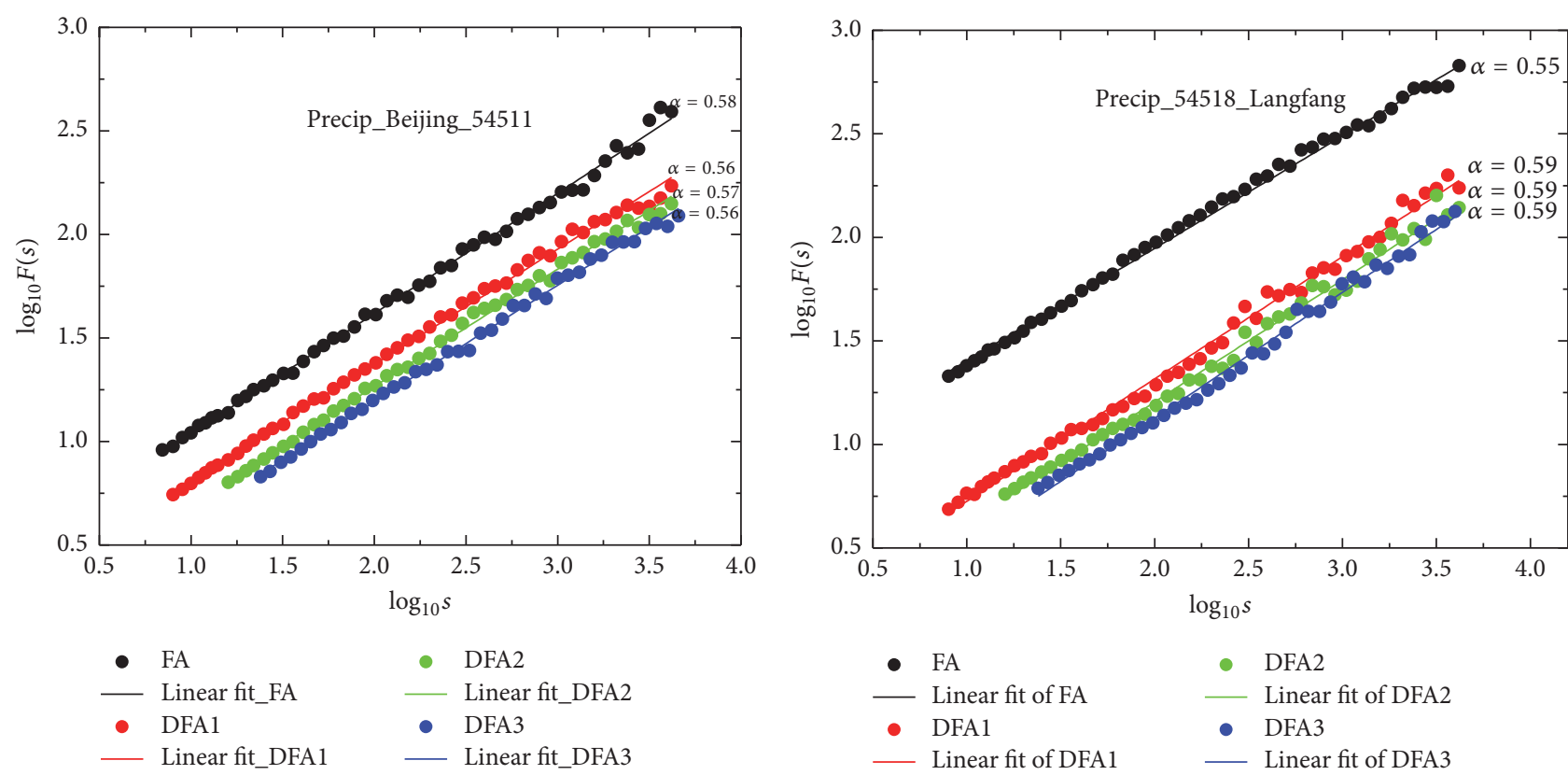

(a)
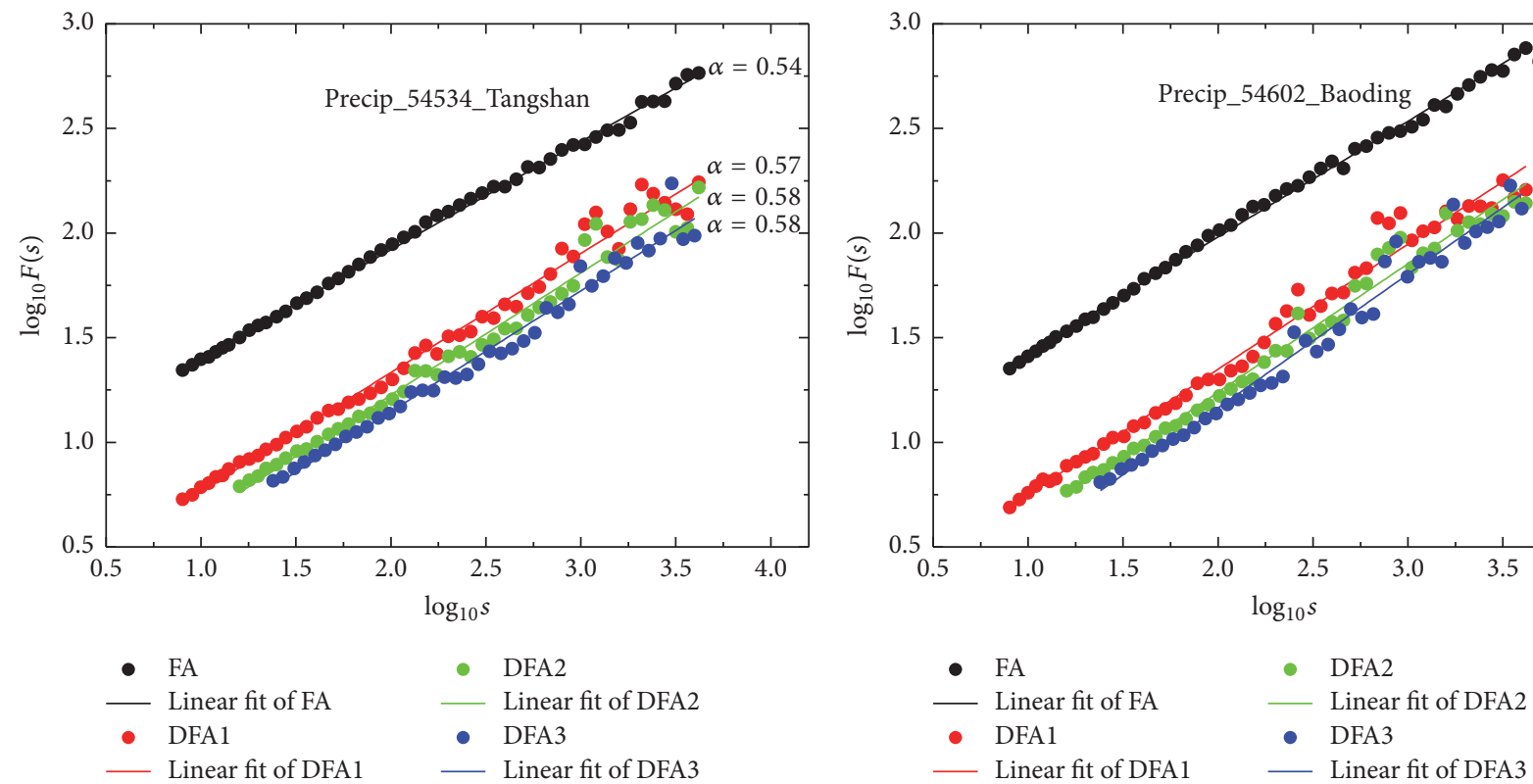

- FA

- DFA2

- Linear fit of FA

- Linear fit of DFA2

- DFA1

- DFA3

(d)

FIGURE 4: Log-log plots of power law relationship in precipitation anomaly records using FA and DFA1-3 (black, red, green, and blue solid circles, resp.) at four stations. (a) Beijing. (b) Langfang. (c) Tangshan. (d) Baoding. Solid lines are linear fits.

than that in temperature. This indicates increasing extreme events occurrence for precipitation anomaly time series.

The changes of the slopes for temperature and precipitation time series in Beijing are almost consistent when the annual cycles are eliminated by employing DFA1-3. So we further investigate the LRCs of temperature and precipitation using the DFA2 method. The long range power law relationships of $\log -\log$ plots between the fluctuation $F(s)$ and the window scale $s$ are given in Figure 7. For temperature records, the value of scaling exponent $\alpha=0.66$ above one month is consistent with that of the earlier finding [27]. However, the slope below one month $\alpha=0.91$ is in the extremely high level, which indicates there is the trend effect of local climate change. For precipitation records, the value 

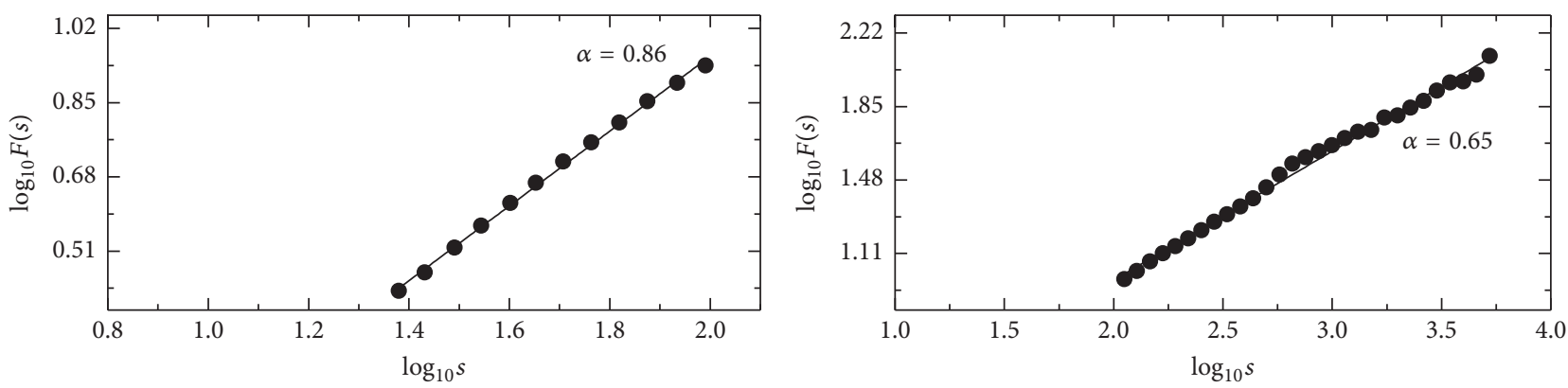

- DFA3

- DFA3

- Linear fit
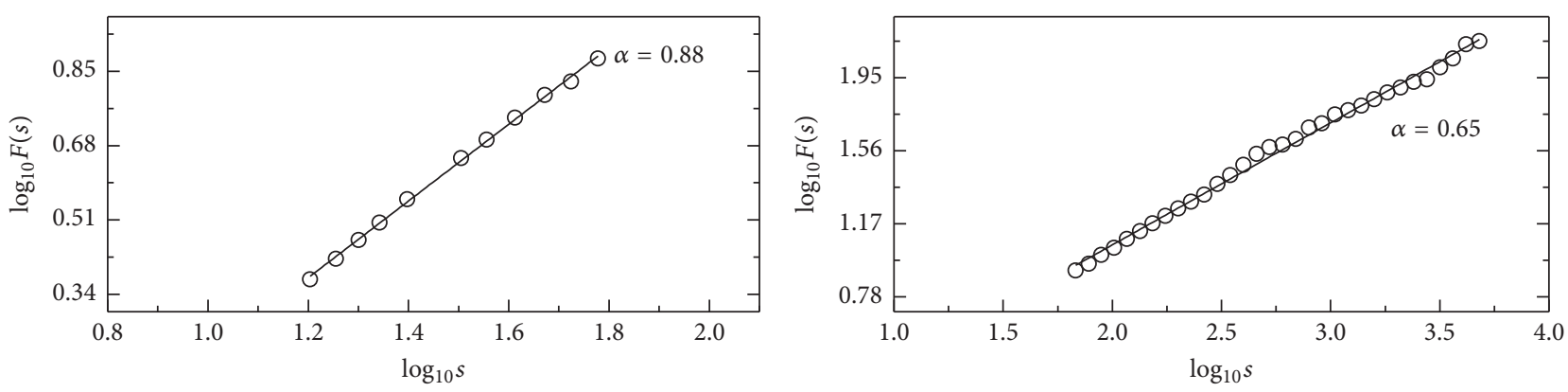

O DFA2

— Linear fit

O DFA2

— Linear fit
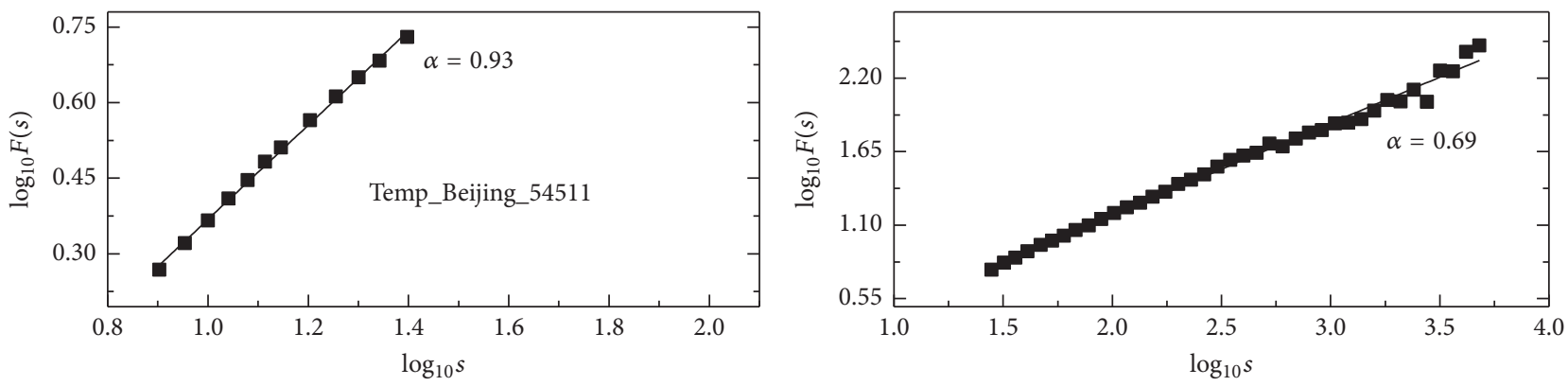

- DFA1

- DFA1

- Linear fit

— Linear fit

FIGURE 5: Log-log plots of power law relationship between the fluctuation $F(s)$ and piecewise time window $s$ for temperature time series in Beijing using DFA1-3 (square, hollow circle, and solid circle, resp.). Solid lines are linear fits.

of scaling exponent $\alpha=0.57$ shows weak LRCs for all time scales. Moreover, LRCs of precipitation records are not affected by local influencing factors.

\section{Conclusion and Discussions}

Many studies are on the scaling behaviors of temperature or precipitation, but few studies are on the difference of scaling properties between temperature and precipitation. Moreover, traditional approaches cannot detect natural variability due to nonstationarity of climate variables. In this letter, longterm daily air temperature and precipitation records of four weather stations over China are investigated by applying the
FA and different orders DFA methods. The results show that there are LRCs of temperature and precipitation records for all weather stations.

Different characteristics of LRCs are found after resampling the data records many times through a 95\% significance test. LRCs of temperature decrease with the increase of time window by employing different orders DFA. However, LRCs of precipitation records are not obvious across all time scales. This may be caused by the factors of complexity and discontinuity of precipitation. The value of scaling exponent for temperature records is significantly higher than that for precipitation records, which implies that there are stronger LRCs for temperature over China. 


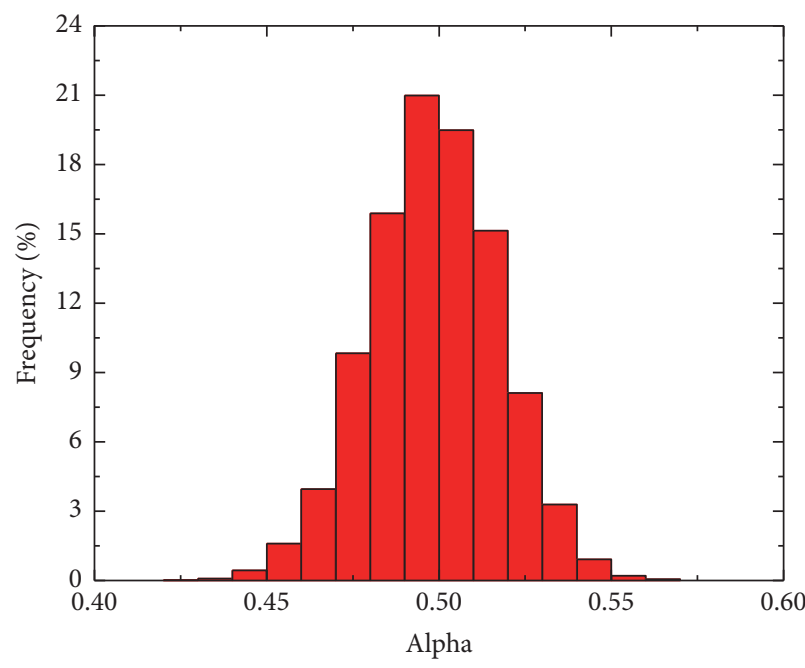

Frequency of Temp_DFA2_Beijing_54511

(a)

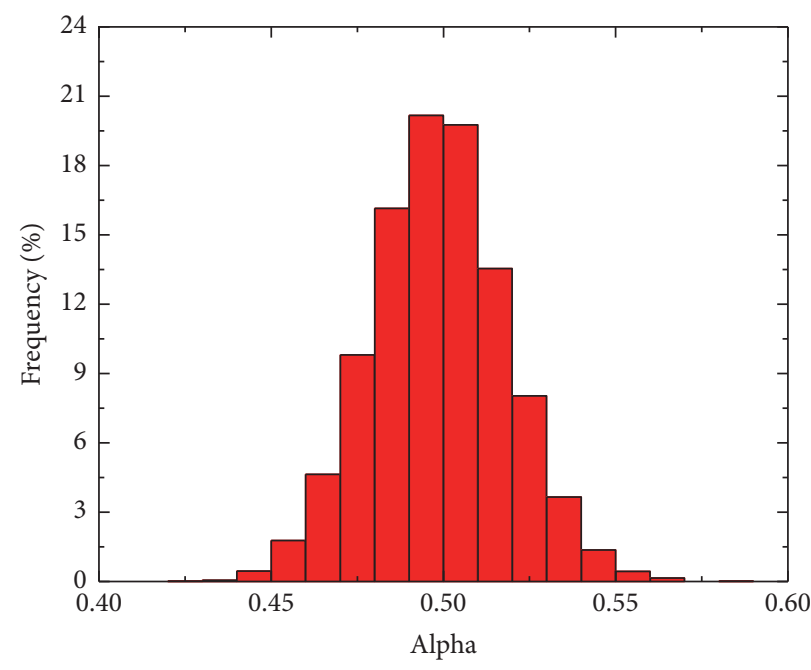

Frequency of Precip_DFA2_Beijing_54511

(b)

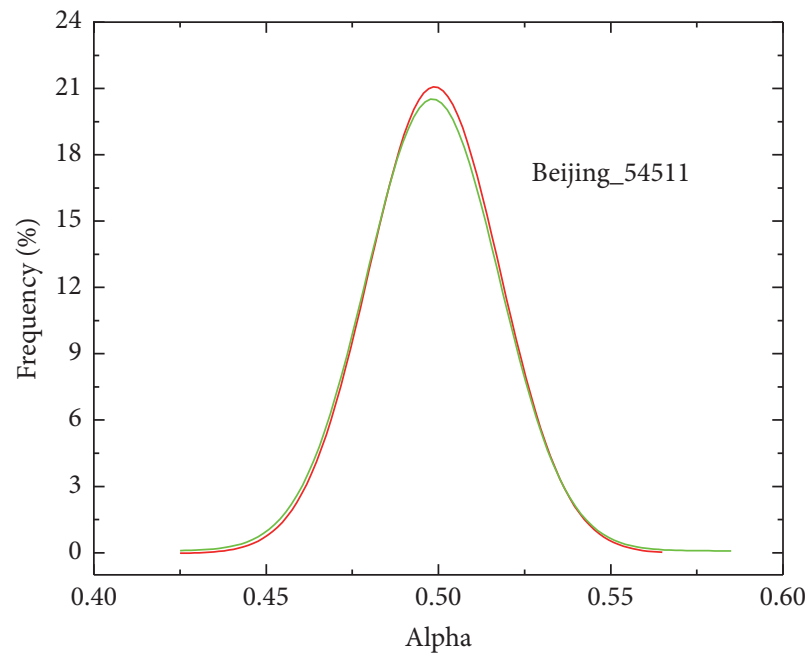

Gauss_fit_shuffled_Temp_DFA2

Gauss_fit_shuffled_Precip_DFA2

(c)

Figure 6: The frequency distributions of the scaling exponent using DFA2 after shuffling 10000 times in Beijing. (a) Temperature. (b) Precipitation. (c) Gauss fits of temperature and precipitation.

In addition, there are similar slopes for temperature and precipitation records by comparing different orders DFA1-3. The value in DFA-exponent for temperature time series is pretty higher at short time scales than that at long time scales, which implies the LRCs can be affected by some local trends, while precipitation records show similar slopes across all time scales. The scaling behaviors of the temperature and precipitation anomaly records might be affected by many different factors at different time scales. It is necessary to perform more studies on the difference of scaling behaviors between temperature and precipitation in the future.

\section{Conflicts of Interest}

The authors declare that there are no conflicts of interest regarding the publication of this paper.

\section{Acknowledgments}

The authors would like to acknowledge the Introducing Talent of NUIST (2014r18; 2013x031), the National Natural Science Foundation of China (11402124), the Natural Science Foundation of Jiangsu Province (BK20140985), the Natural Science Foundation of Jiangsu Higher Education Institutions 




FIGURE 7: Log-log plots of power law relationship for temperature and precipitation time series in Beijing using DFA2 (black, green, and blue solid circles, resp.). Solid lines are linear fits.

of China (14KJB130002), and the Priority Academic Program Development of Jiangsu Higher Education Institutions (PAPD).

\section{References}

[1] D. R. Easterling, B. Horton, P. D. Jones et al., "Maximum and minimum temperature trends for the globe," Science, vol. 277, no. 5324, pp. 364-367, 1997.

[2] C.-K. Peng, S. V. Buldyrev, S. Havlin, M. Simons, H. E. Stanley, and A. L. Goldberger, "Mosaic organization of DNA nucleotides," Physical Review E: Statistical, Nonlinear, and Soft Matter Physics, vol. 49, no. 2, pp. 1685-1689, 1994.

[3] P. Talkner and R. O. Weber, "Power spectrum and detrended fluctuation analysis: Application to daily temperatures," Physical Review E, vol. 62, p. 150, 2000.

[4] R. B. Govindan, D. Vyushin, A. Bunde, S. Brenner, S. Havlin, and H. Schellnhuber, "Global climate models violate scaling of the observed atmospheric variability," Physical Review Letters, vol. 89 , no. 2, 2002.

[5] K. Fraedrich and R. Blender, "Scaling of atmosphere and ocean temperature correlations in observations and climate models," Physical Review Letters, vol. 10, p. 90, 2003.

[6] M. L. Kurnaz, "Application of detrended fluctuation analysis to monthly average of the maximum daily temperatures to resolve different climates," Fractals, vol. 12, no. 4, pp. 365-373, 2004.

[7] A. Király and I. M. Jánosi, "Detrended fluctuation analysis of daily temperature records: geographic dependence over Australia. Meteorol," Meteorology and Atmospheric Physics, vol. 88, pp. 119-128, 2005.

[8] C. A. Varotsos, M. N. Efstathiou, and A. P. Cracknell, "On the scaling effect in global surface air temperature anomalies," Atmospheric Chemistry and Physics, vol. 13, no. 10, pp. 52435253, 2013.
[9] K. Ivanova, M. Ausloos, E. E. Clothiaux, and T. P. Ackerman, "Break-up of stratus cloud structure predicted from nonBrownian motion liquid water and brightness temperature fluctuations," EPL (Europhysics Letters), vol. 52, no. 1, pp. 40-46, 2000.

[10] R. B. Govindan and H. Kantz, "Long-term correlations and multifractality in surface wind speed," EPL, vol. 68, p. 184, 2004.

[11] X. Chen, G. Lin, and Z. Fu, "Long-range correlations in daily relative humidity fluctuations: A new index to characterize the climate regions over China," Geophysical Research Letters, Article ID L07804, 2007.

[12] G. Lin, X. Chen, and Z. Fu, "Temporal-spatial diversities of long-range correlation for relative humidity over China," Physica A: Statistical Mechanics and its Applications, vol. 383, no. 2, pp. 585-594, 2007.

[13] C. Varotsos, J. Ondov, and M. Efstathiou, "Scaling properties of air pollution in Athens, Greece and Baltimore, Maryland," Atmospheric Environment, vol. 39, no. 22, pp. 4041-4047, 2005.

[14] C. Varotsos, M. Efstathiou, and C. Tzanis, "Scaling behaviour of the global tropopause," Atmospheric Chemistry and Physics, vol. 9, no. 2, pp. 677-683, 2009.

[15] C. Varotsos, "Power-law correlations in column ozone over Antarctica," International Journal of Remote Sensing, vol. 26, no. 16, pp. 3333-3342, 2005.

[16] M. Luo, Y. Leung, Y. Zhou, and W. Zhang, "Scaling behaviors of global sea surface temperature," Journal of Climate, vol. 28, no. 8, pp. 3122-3132, 2015.

[17] L. Jiang, X. Zhao, and L. Wang, "Long-Range Correlations of Global Sea Surface Temperature," PloSone, vol. 11, Article ID e0153774, 2016.

[18] M. N. Efstathiou, C. Tzanis, A. P. Cracknell, and C. A. Varotsos, "New features of land and sea surface temperature anomalies," International Journal of Remote Sensing, vol. 32, no. 11, pp. 32313238, 2011.

[19] L. Jiang, N. Yuan, Z. Fu, D. Wang, X. Zhao, and X. Zhu, "Subarea characteristics of the long-range correlations and the index $\chi$ for daily temperature records over China," Theoretical and Applied Climatology, vol. 109, pp. 261-270, 2012.

[20] L. Jiang, N. Li, Z. Fu, and J. Zhang, "Long-range correlation behaviors for the 0 - $\mathrm{cm}$ average ground surface temperature and average air temperature over China," Theoretical and Applied Climatology, vol. 119, pp. 25-31, 2015.

[21] L. Jiang, N. Li, and X. Zhao, "Scaling behaviors of precipitation over China," Theoretical and Applied Climatology, vol. 18, pp. 18, 2015.

[22] N. Yuan, Z. Fu, and J. Mao, "Different scaling behaviors in daily temperature records over China," Physica A: Statistical Mechanics and its Applications, vol. 389, pp. 4087-4095, 2010.

[23] L. Jiang, J. Zhang, X. Liu, and F. Li, "Multi-fractal scaling comparison of the air temperature and the surface temperature over China," Physica A: Statistical Mechanics and its Applications, vol. 462, pp. 783-792, 2016.

[24] A. Bunde, S. Havlin, J. W. Kantelhardt, T. Penzel, J.-H. Peter, and K. Voigt, "Correlated and uncorrelated regions in heart-rate fluctuations during sleep," Physical Review Letters, vol. 85, no. 17, pp. 3736-3739, 2000.

[25] J. W. Kantelhardt, S. A. Zschiegner, E. Koscielny-Bunde, S. Havlin, A. Bunde, and H. E. Stanley, "Multifractal detrended fluctuation analysis of nonstationary time series," Physica A: Statistical Mechanics and its Applications, vol. 316, no. 1-4, pp. 87-114, 2002. 
[26] K. Hu, P. C. Ivanov, Z. Chen, P. Carpena, and H. Eugene Stanley, "Effect of trends on detrended fluctuation analysis," Physical Review E: Statistical, Nonlinear, and Soft Matter Physics, vol. 64, no. 1, 2001.

[27] E. Koscielny-Bunde, A. Bunde, S. Havlin, H. Eduardo Roman, Y. Goldreich, and H.-J. Schellnhuber, "Indication of a universal persistence law governing atmospheric variability," Physical Review Letters, vol. 81, no. 3, pp. 729-732, 1998. 

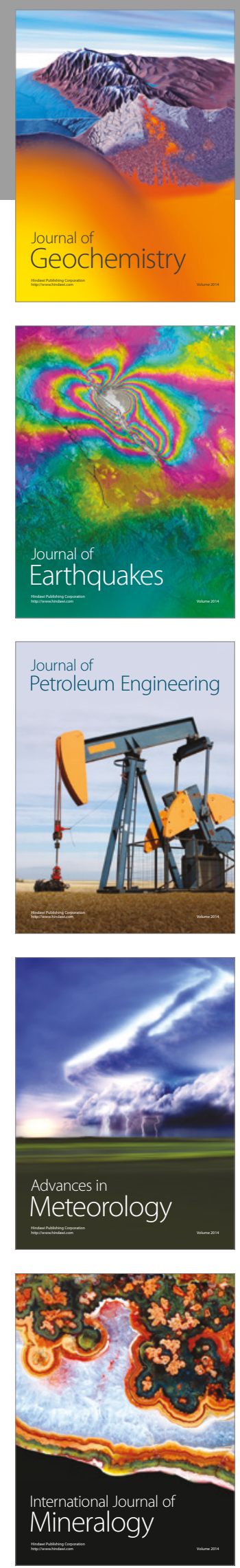
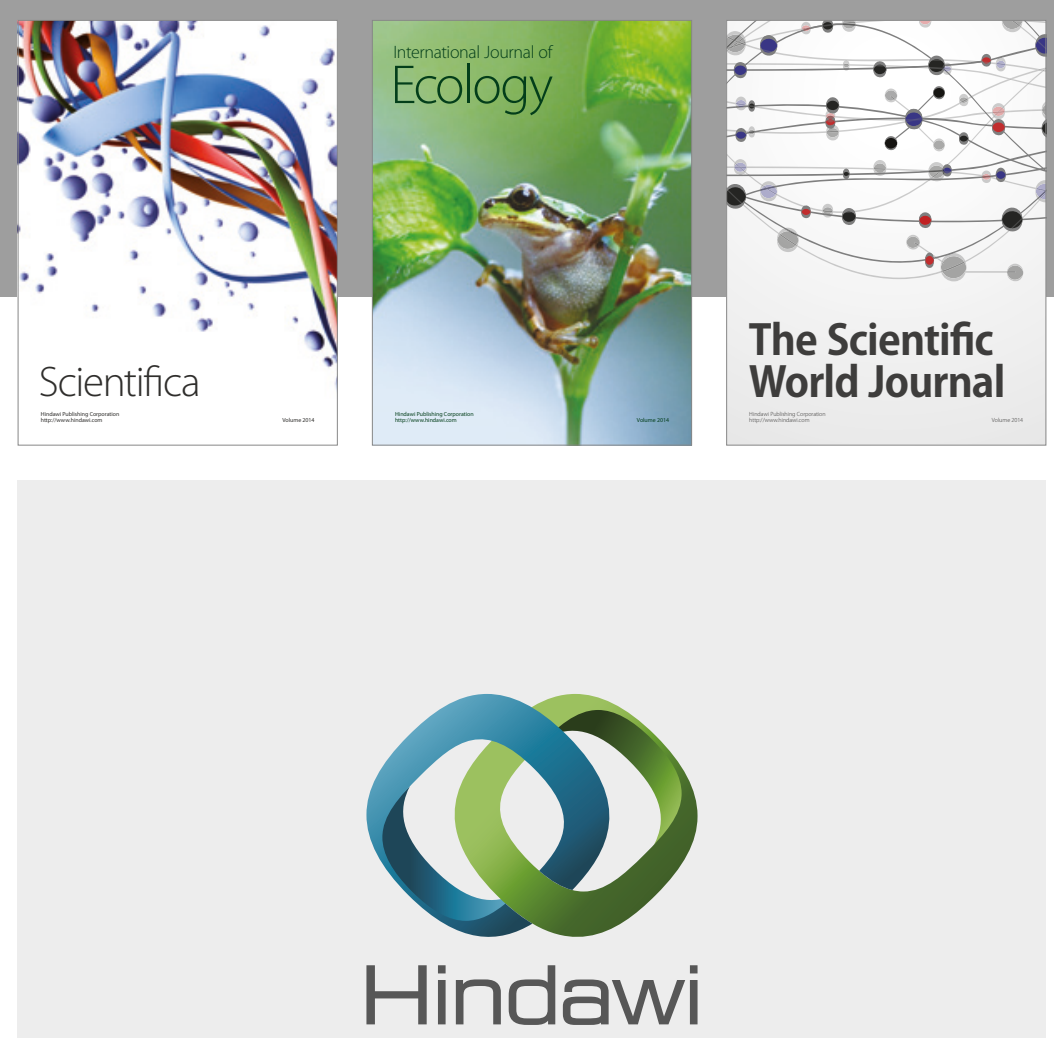

Submit your manuscripts at

https://www.hindawi.com
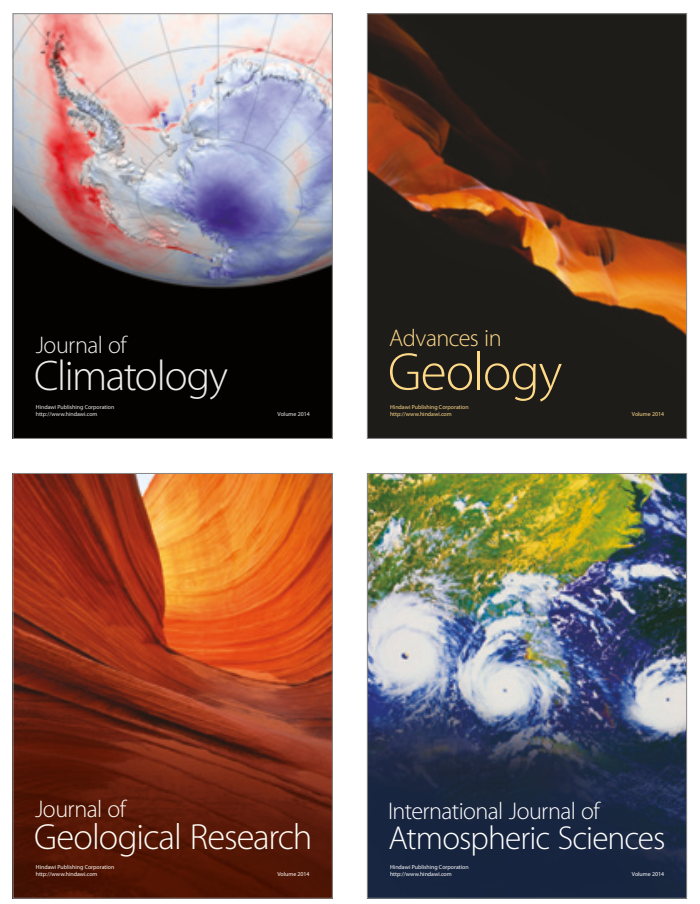

The Scientific

World Journal
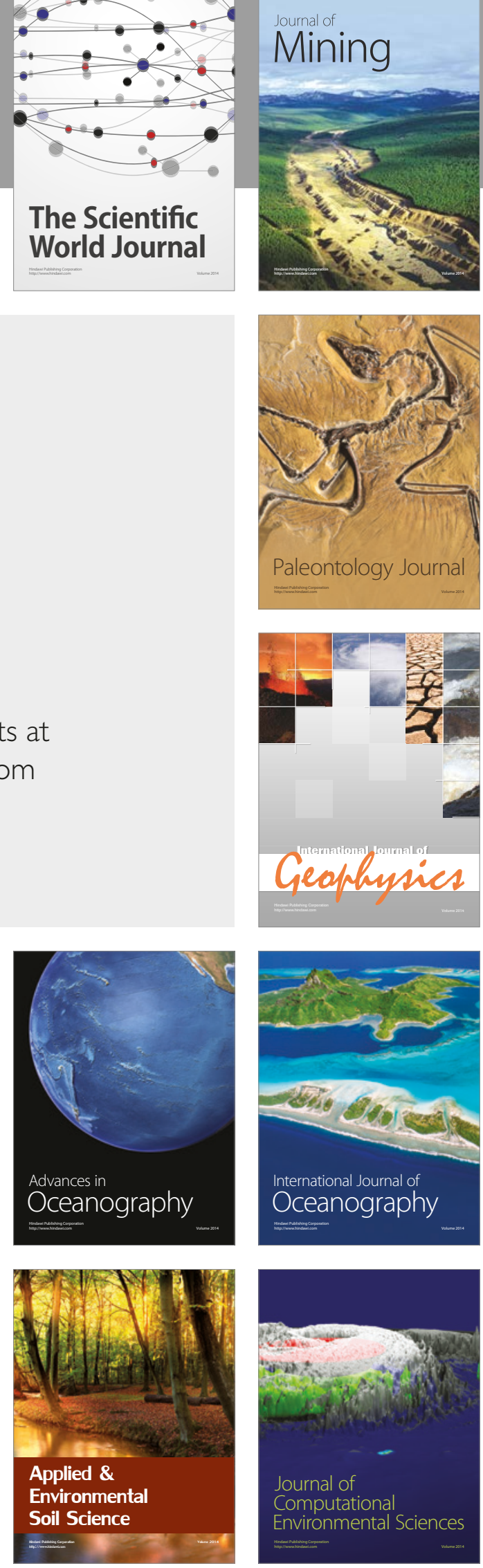